\title{
BMJ Open Acupuncture for the treatment of chronic obstructive pulmonary disease: a protocol of a systematic review
}

\author{
Tae-Young Choi, ${ }^{1}$ Ji Hee Jun, ${ }^{1}$ Jun-Young Choi, ${ }^{2}$ Jong-In Kim, ${ }^{3}$ Myeong Soo Lee, ${ }^{1}$ \\ Edzard Ernst ${ }^{4}$
}

To cite: Choi T-Y, Jun JH, Choi J-Y, et al. Acupuncture for the treatment of chronic obstructive pulmonary disease: a protocol of a systematic review. BMJ Open 2014;4:e004590.

doi:10.1136/bmjopen-2013004590

- Prepublication history and additional material is available. To view please visit the journal (http://dx.doi.org/ 10.1136/bmjopen-2013004590).

Received 1 December 2013 Revised 26 March 2014 Accepted 27 March 2014
CrossMark

For numbered affiliations see end of article.

Correspondence to Dr Myeong Soo Lee; drmslee@gmail.com

\section{ABSTRACT}

Introduction: This review aims to evaluate the efficacy of acupuncture in the treatment of chronic obstructive pulmonary disease (COPD).

Methods and analysis: 14 databases will be searched from their inception. These include PubMed, AMED, EMBASE, the Cochrane Library, seven Korean medical databases (Korean Studies Information Service System, DBPIA, Oriental Medicine Advanced Searching Integrated System, Research Information Service System, KoreaMed, The Town Society of Science Technology and the Korean National Assembly Library), three Chinese Databases (China National Knowledge Infrastructure Database (CNKI), the Chongqing VIP Chinese Science and Technology Periodical Database (VIP), and the Wanfang Database). Only randomised clinical trials (RCTs) using acupuncture for COPD will be considered. The selection of the studies, data abstraction and validation will be performed independently by two researchers. Methodological quality will be assessed with the Cochrane risk of bias.

Dissemination: The systematic review will be published in a peer-reviewed journal. The review will also be disseminated electronically and in print. Updates of the review will be conducted to inform and guide the healthcare practice and policy.

Trial registration number: PROSPERO 2013: CRD42013004824.

\section{INTRODUCTION}

\section{Description of the condition}

Chronic obstructive pulmonary disease (COPD) is a slowly progressive disease characterised by airflow limitation and the gradual loss of lung function that is not fully reversible. ${ }^{1}$ COPD includes emphysema and chronic bronchitis, and the main symptoms include cough and breathlessness. This disease is predominantly caused by smoking. ${ }^{2}$ COPD is a serious public health problem; according to the WHO, an estimated 64 million people worldwide currently suffer from moderate to severe COPD. ${ }^{3}$ In fact, the WHO ranked COPD as the fifth leading cause

\section{Strengths and limitations of this study}

- The strength of this systematic review is its extensive, unbiased search of various databases without a language restriction.

- The trial screening, data extraction and assessing risk of bias will be conducted independently by two of the authors.

- Our systematic review may pertain to the potential incompleteness of the evidence reviewed including publication and location bias, poor quality of the primary data and poor reporting of results.

of death worldwide, and it is estimated that COPD will be ranked as the third leading cause of death by $2030 .{ }^{45}$ COPD also places a significant burden on healthcare systems, and a loss in health-related quality of life is observed in many patients. ${ }^{6}$ Therefore, appropriate therapies are necessary to address this disease.

\section{Description of the intervention}

In China, Traditional Chinese Medicine, as an auxiliary therapy to Western medicine, was extensively employed for the treatment of stable COPD. ${ }^{7}$ Acupuncture is a popular treatment for COPD in China. ${ }^{8}$ The most common treatment for pain in patients with COPD was acupuncture/transcutaneous electrical nerve stimulation compared with physiotherapy in the Norwegian general population. ${ }^{9}$ Proponents argue that acupuncture is effective at relieving symptoms, reducing the incidence of COPD exacerbations and improving quality of life, and that it is associated with fewer adverse effects than conventional approaches to COPD. ${ }^{10}$

\section{How the intervention might work}

Acupuncture may help relieve COPD by reducing bronchial immune-mediated inflammation $^{11}$ and reducing inflammation in general 
by promoting release of vascular and immunomodulatory factors. ${ }^{12}$ However, the reliable evidence is unclear. ${ }^{13}$

\section{Why it is important to this review}

Response to treatment may differ with the pathophysiological variation between stable and acute disease. Acute exacerbations of COPD (AECOPD) are defined by acute, excessive increases in dyspnoea, cough and/or sputum, and are often associated with bacterial infection, neutrophilic inflammation and specific immune responses. ${ }^{14}$

\section{Objectives}

This review aims at systematically evaluating the evidence of acupuncture for treating AECOPD and stable COPD from RCTs.

\section{METHODS}

Study registration

The protocol of this systematic review has been registered on PROSPERO 2013 (registration number: CRD42013004824). ${ }^{15}$ This systematic review protocol was conducted and reported using the Preferred Reporting Items for Systematic Reviews and Meta-Analyses (PRISMA) statement guidelines. ${ }^{16}$

\section{Data sources}

The following databases were searched from their inception: PubMed, AMED, EMBASE, The Cochrane Library, seven Korean Medical Databases (Korean Studies Information Service System, DBPIA, Oriental Medicine Advanced Searching Integrated System, Research Information Service System, KoreaMed, The Town Society of Science Technology and the Korean National Assembly Library), and the China National Knowledge Infrastructure Database (CNKI), the Chongqing VIP Chinese Science and Technology Periodical Database (VIP), and Wanfang Database. Articles identified through reference lists of included studies and relevant systematic reviews will be considered for inclusion based on their title. Our search strategy will include main keywords 'acupuncture' and 'chronic obstructive pulmonary disease' (see online supplement 1). Study selection will be documented and summarised in a PRISMA compliant flow chart (figure 1).

\section{Eligibility criteria}

Population

We will include populations with a diagnosis of COPD. We will only include studies in which an external set of criteria had been used to screen participants for the condition (eg, criteria from the Global Initiative for Obstructive Lung Disease (GOLD), ${ }^{1}$ American Thoracic Society (ATS), ${ }^{17}$ British Thoracic Society (BTS) ${ }^{18}$ or Group of Chronic Obstructive Pulmonary Diseases/the Branch of Respiratory Diseases/Chinese Medical Association ${ }^{19-21}$ ).
Patients who are clinically stable and do not show evidence of an exacerbation 1 month prior to study entry will be included. Patients at stable state and with exacerbations of COPD will be included. Patients with significant diseases other than COPD, including a diagnosis of asthma, cystic fibrosis, bronchiectasis or other lung diseases, will be excluded.

\section{Interventions}

Studies that evaluated any type of invasive acupuncture will be included. The treatments considered have to involve needle insertion at acupuncture points, pain points or trigger points and had to be described as acupuncture. Studies investigating other methods of stimulating acupuncture points without needle insertion (eg, acupressure, pressed studs, laser stimulation or transcutaneous electrical stimulation) will be excluded. Control interventions in controlled studies may include treatments such as general care, sham treatment (interventions mimicking 'true' acupuncture/true treatment but deviating in at least one aspect considered important by acupuncture theory, such as skin penetration or correct point location), waiting list care or other treatment (eg, relaxation and physical therapies). We will also include trials that compared acupuncture plus another active treatment versus that other active treatment alone. Thus, we will include all pragmatic trials that compared acupuncture with any other treatments (eg, drugs, exercise, education, etc). Because our objective is to evaluate the effects of acupuncture compared with non-acupuncture controls, we will exclude RCTs in which one form of acupuncture was compared with another form of acupuncture.

\section{Outcome measures}

\section{Primary outcomes}

1. Treatment efficacy: the number of patients whose COPD symptoms improved.

2. Quality of life: measured using a validated questionnaire, for example, St. George's Respiratory Questionnaire $(\mathrm{SGRQ})^{22}$ or the Chronic Respiratory Disease Questionnaire (CRDQ or CRQ). ${ }^{23}$

3. Exacerbations ${ }^{24}$ : frequency of exacerbations, time to first exacerbation, severity and duration of exacerbations.

\section{Secondary outcomes}

1. Pulmonary function: change in forced expiratory volume in $1 \mathrm{~s}$ and change in forced ventilatory capacity $^{25}$ (trough, peak and average) and other measures of pulmonary function.

2. Dyspnoea scores, for example, the Borg scale score, ${ }^{26}$ the visual analogue scale (VAS), ${ }^{27}$ the Medical Research Council (MRC) dyspnoea scale ${ }^{28}$ components of the COPD assessment test (CAT) ${ }^{29}$

3. Anxiety on a $10 \mathrm{~cm}$ VAS. ${ }^{27}$

4. Exercise tolerance: for example, 6 min walk test, ${ }^{30}$ shuttle walk test. ${ }^{31}$

5. Adverse events.

6. Participant withdrawal. 


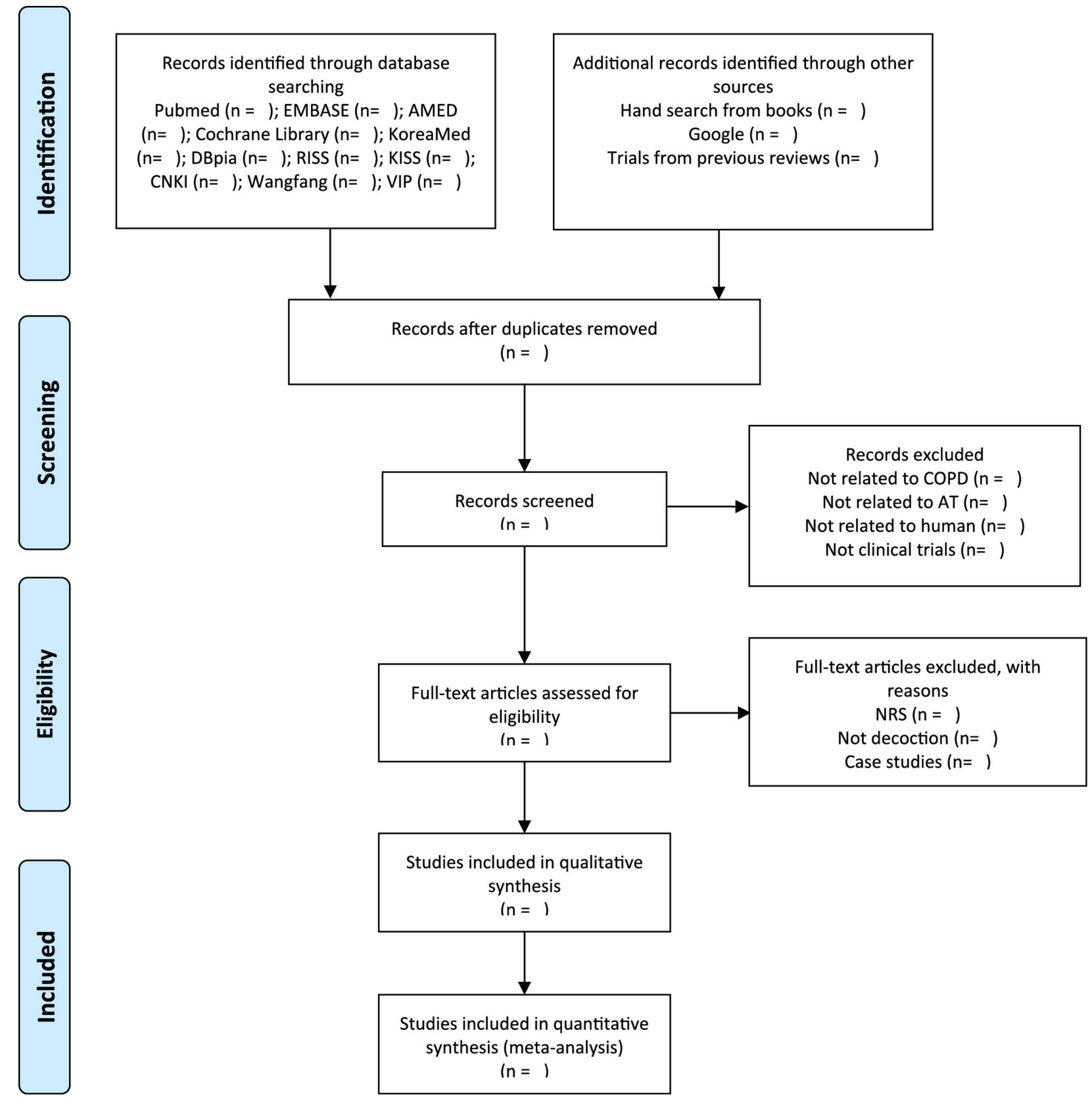

Figure 1 Flow diagram of the trial selection process. AT, acupuncture; COPD, chronic obstructive pulmonary disease; NRS, non-randomised studies.

\section{Study design}

Only RCTs will be included. Observational, cohort, case-control, case series, qualitative studies, uncontrolled trials and laboratory studies were excluded.

\section{Data collection and analysis}

Data extraction

All articles will be read by two independent reviewers who extract data from the articles according to predefined criteria. The extracted data will include the authors, year of publication, country, study size, age and gender of the participants, acupuncture intervention, control intervention, main outcomes and adverse effects. The extracted data will be tabulated (see online supplement 2) for further analysis. Details regarding the acupuncture and control interventions will be extracted on the basis of the revised Standards for Reporting Interventions in Clinical Trials of Acupuncture $\left(\right.$ STRICTA) ${ }^{32}$ (see online supplement 3$)$.

\section{Risk of bias assessment}

Quality assessment will be performed using the tool for 'risk of bias' from the Cochrane Handbook for Systematic Reviews of Interventions (see online supplement 4) ${ }^{33}$ The following characteristics will be assessed: (1) Was the allocation sequence adequately generated? (2) Was the allocation adequately concealed? (3) Was knowledge of the allocated interventions adequately 
presented during the study? (4) Were incomplete outcome data adequately addressed? (5) Were the study reports free of the suggestion of selective outcome reporting? and (6) Was the study free of other problems that could introduce a risk of bias? This review used ' $\mathrm{L}$, $\mathrm{U}$ and $\mathrm{H}$ ' as keys for these judgements; where 'Low' (L) indicated a low risk of bias, 'Unclear' (U) indicated that the risk of bias was uncertain, and 'High' $(\mathrm{H})$ indicated a high risk of bias. Disagreements will be resolved by discussion between all authors.

\section{Data synthesis}

All statistical analyses will be conducted using the Cochrane Collaboration's software program, Review Manager (RevMan), V.5.1 for Windows (Copenhagen, The Nordic Cochrane Center). Differences between the intervention and control groups will be assessed. In the analysis of clinical efficacy, count data will be assessed in terms of risk ratios, and continuous data will be assessed in terms of mean difference (MD). Count data and continuous variables will be expressed as efficacy values with $95 \%$ CIs. In cases of outcome variables with different scales, the standard mean difference will be used instead of the weighted MD. If the meta-analyses exhibit heterogeneity (defined as results of tests of heterogeneity that indicate that $\mathrm{p}<0.1$ and $\mathrm{I}^{2} \geq 50 \%$ ), then a random effects model will be used to assess combined efficacy values; otherwise, fixed effects models were used for these assessments. Publication bias will be assessed using funnel plots and Egger's regression method. ${ }^{34}$ If missing data are detected, we will request any missing or incomplete information from the original study investigators. Subgroup analysis will be conducted according to different control interventions (sham acupuncture vs conventional medication), the type of acupuncture (Chinese vs Western; standardised acupuncture vs individually adapted acupuncture points), type of stimulation (manual vs electric), treatment frequency (less than 14 vs more than 14), the design of the trial (acupuncture vs sham acupuncture; acupuncture vs conventional medication; acupuncture combined conventional medication vs conventional medication). Sensitivity analysis will be performed to evaluate the robustness of the meta-analysis results. Consistent with other meta-analyses and meta-regressions, ${ }^{35}$ the primary quality measure will be a binary measure of allocation concealment. ${ }^{36}$ Therefore, the risk of bias assessment for included studies will be summarised in a table, and the results and implications will be critically discussed.

\section{DISCUSSION}

Until now, no systematic reviews have examined the use of acupuncture in the treatment of COPD. This systematic review will provide a detailed summary of the current evidence related to the effectiveness of acupuncture in treating the symptoms of patients with COPD. This evidence will be useful to practitioners, patients and health policy-makers regarding the use of acupuncture in COPD treatment.

\section{Author affiliations}

${ }^{1}$ Medical Research Division, Korea Institute of Oriental Medicine, Daejeon, South Korea

${ }^{2}$ Department of Korean Medical Science, School of Korean Medicine, Pusan National University, Yangsan, South Korea

${ }^{3}$ Department of Acupuncture and Moxibustion, College of Korean Medicine, Kyung Hee University, Seoul, South Korea

${ }^{4}$ Complementary Medicine, Peninsula Medical School, University of Exeter, Exeter, UK

Contributors T-YC and MSL conceived the study, developed the criteria and searched the literature, performed data analysis and wrote the protocol. JHJ assisted in searching the Chinese literature and extracting data. J-YC and J-IK wrote the introduction of this protocol. EE advised on the protocol design and revised the manuscript. All authors read and approved the final manuscript.

\section{Competing interests None.}

Provenance and peer review Not commissioned; externally peer reviewed.

Open Access This is an Open Access article distributed in accordance with the Creative Commons Attribution Non Commercial (CC BY-NC 3.0) license, which permits others to distribute, remix, adapt, build upon this work noncommercially, and license their derivative works on different terms, provided the original work is properly cited and the use is non-commercial. See: http:// creativecommons.org/licenses/by-nc/3.0/

\section{REFERENCES}

1. Global initiative for chronic obstructive lung disease. Global strategy for the diagnosis, management, and prevention of chronic obstructive pulmonary disease: updated 2013. http://www.goldcopd. org (accessed Nov 2013).

2. National Institute for Health and Care Excellence. Chronic obstructive pulmonary disease (update): full guideline 2010. http:// guidanceniceorguk/CG101/Guidance/pdf/English (accessed Nov 2013)

3. World Health Organization. Chronic obstructive pulmonary disease (COPD). http://wwwwhoint/mediacentre/factsheets/fs315/en/ indexhtml (accessed Nov 2013).

4. Murray CJ, Lopez AD. Alternative projections of mortality and disability by cause 1990-2020: Global Burden of Disease Study. Lancet 1997;349:1498-504.

5. World Health Organization. Chronic obstructive pulmonary disease (COPD). http://wwwwhoint/respiratory/copd/en/ (accessed Nov 2013).

6. Menzin J, Boulanger L, Marton J, et al. The economic burden of chronic obstructive pulmonary disease (COPD) in a US Medicare population. Respir Med 2008;102:1248-56.

7. Guo YM, Tong J, Yao H. Effect of acupuncture on respiratory function of stable chronic obstructive pulmonary disease. J Guangzhou Uni Tradit Chin Med 2013;30:658-62.

8. Jiang YL, Wang HF, Li JS. Research comment on the treatment of respiratory failure of acute exacerbation of chronic obstructive pulmonary disease by Chinese medicine. China $J$ Chin Med 2012;27:670-72.

9. Bentsen SB, Rustøen T, Miaskowski C. Prevalence and characteristics of pain in patients with chronic obstructive pulmonary disease compared to the Norwegian general population. $J$ Pain 2011;12:539-45.

10. Suzuki M, Namura K, Ohno $\mathrm{Y}$, et al. Combined standard medication and acupuncture for COPD: a case series. Acupunct Med 2012;30:96-102.

11. Carneiro ER, Carneiro CR, Castro MA, et al. Effect of electroacupuncture on bronchial asthma induced by ovalbumin in rats. J Altern Complement Med 2005;11:127-34.

12. Zijlstra FJ, van den Berg-de Lange I, Huygen FJ, et al. Anti-inflammatory actions of acupuncture. Mediators Inflamm 2003;12:59-69.

13. Gibson D, Bruton A, White P. Acupuncture for respiratory disorder: what's the point? Expert Rev Respir Med 2010;4:29-37.

14. Rabe KF, Hurd S, Anzueto A, et al. Global strategy for the diagnosis, management, and prevention of chronic obstructive 
pulmonary disease: GOLD executive summary. Am J Respir Crit Care Med 2007;176:532-55.

15. PROSPERO. PROSPERO International prospective register of systematic reviews. http://www.crd.york.ac.uk/PROSPERO/display record.asp?ID=CRD42013004824

16. Liberati A, Altman DG, Tetzlaff J, et al. The PRISMA statement for reporting systematic reviews and meta-analyses of studies that evaluate health care interventions: explanation and elaboration. Ann Intern Med 2009;151:W-65-94.

17. American Thoracic Society. Standards for the diagnosis and care of patients with chronic obstructive pulmonary disease. Am J Respir Crit Care Med 1995;152(5 Pt 2):S77-121.

18. COPD Guidelines Group of the Standards of Care Committee of the BTS. BTS Guidelines for the management of chronic obstructive pulmonary disease. Thorax 1997;52:S7-15.

19. Branch of Respiratory Diseases/Chinese Medical Association. Diagnostic and therapeutic standards of chronic obstructive pulmonary disease (COPD) (draft). Zhong Hua Jie He He Hu Xi Za Zhi 1997;20:199-203.

20. Group of Chronic Obstructive Pulmonary Disease/Branch of Respiratory Diseases/Chinese Medical Association. Guidance of diagnosis and treatment of chronic obstructive pulmonary disease. Zhong Hua Jie He He Hu Xi Za Zhi 2002;25:453-60.

21. Group of Chronic Obstructive Pulmonary Disease/Branch of Respiratory Diseases/Chinese Medical Association. Guidance of diagnosis and treatment of chronic obstructive pulmonary disease (reversed edition in 2007). Zhong Hua Jie He He Hu Xi Za Zhi 2007;30:8.

22. Jones PW, Quirk FH, Baveystock CM, et al. A self-complete measure of health status for chronic airflow limitation: the St George's Respiratory Questionnaire. Am Rev Respir Dis 1992;145:1321-7.

23. Schünemann HJ, Puhan M, Goldstein R, et al. Measurement properties and interpretability of the chronic respiratory disease questionnaire (CRQ). COPD 2005;2:81-9.

24. Steer J, Gibson GJ, Bourke SC. Predicting outcomes following hospitalization for acute exacerbations of COPD. QJM 2010;103:817-29.
25. American Thoracic Society. Standardization of spirometry, 1994 update. Am J Respir Crit Care Med 1995;152:1107-36.

26. Borg GA. Psychophysical bases of perceived exertion. Med Sci Sports Exerc 1982;14:377-81.

27. Kindler $\mathrm{CH}$, Harms $\mathrm{C}$, Amsler $\mathrm{F}$, et al. The visual analog scale allows effective measurement of preoperative anxiety and detection of patients' anesthetic concerns. Anesth Analg 2000; 90:706-12.

28. Stenton C. The MRC breathlessness scale. Occup Med (Lond) 2008;58:226-7.

29. Raghavan N, Lam YM, Webb KA, et al. Components of the COPD Assessment Test (CAT) associated with a diagnosis of COPD in a random population sample. COPD 2012;9:175-83.

30. ATS Committee on Proficiency Standards for Clinical Pulmonary Function Laboratories. ATS statement: guidelines for the six-minute walk test. Am J Respir Crit Care Med 2002;166:111-17.

31. Singh SJ, Morgan MD, Scott S, et al. Development of a shuttle walking test of disability in patients with chronic airways obstruction. Thorax 1992;47:1019-24.

32. MacPherson H, Altman DG, Hammerschlag R. Revised STandards for Reporting Interventions in Clinical Trials of Acupuncture (STRICTA): extending the CONSORT statement. PLoS Med 2010;7: e1000261.

33. Higgins JPT, Altman DG, Sterne JAC. Chapter 8: assessing risk of bias in included studies. In: Higgins JPT, Green S. eds Cochrane handbook for systematic reviews of interventions version 5.1.0 (updated March 2011). The Cochrane collaboration, 2011. http:// www.cochrane-handbook.org

34. Egger M, Davey Smith G, Schneider M, et al. Bias in meta-analysis detected by a simple, graphical test. BMJ 1997;315:629-34.

35. Harkness E, MacDonald W, Valderas J, et al. Identifying psychosocial interventions that improve both physical and mental health in patients with diabetes: a systematic review and meta-analysis. Diabetes Care 2010;33:926-30.

36. Hewitt C, Hahn S, Torgerson DJ, et al. Adequacy and reporting of allocation concealment: review of recent trials published in four general medical journals. BMJ 2005;330:1057-8. 